\title{
COVID-19 and Disease-Modifying Anti-rheumatic Drugs
}

\author{
Kristin M. D'Silva ${ }^{1,2,3}$ (D) Zachary S. Wallace ${ }^{1,2,3}$ \\ Accepted: 23 February 2021 / Published online: 24 April 2021 \\ (C) The Author(s), under exclusive licence to Springer Science+Business Media, LLC part of Springer Nature 2021
}

\begin{abstract}
Purpose of Review Patients on disease-modifying anti-rheumatic drugs (DMARDs) remain concerned about potential risks of severe COVID-19 outcomes. Meanwhile, several DMARDs have been proposed as COVID-19 therapies.

Recent Findings In patients with autoimmune diseases, baseline glucocorticoid use is associated with severe COVID-19. While classes of DMARDs (e.g., conventional synthetic, targeted synthetic, and biologic) do not appear to be associated with higher risk, specific medications such as rituximab and sulfasalazine may be associated. Randomized clinical trials (RCTs) show that glucocorticoids reduce mortality in severe COVID-19. RCTs suggest other agents, such as baricitinib, may improve COVID-19 outcomes in certain populations.

Summary Baseline glucocorticoid use raises the risk of severe COVID-19 in patients with autoimmune diseases, but glucocorticoids are an effective treatment for those with severe COVID-19. Further research is needed to inform DMARD management in autoimmune disease patients during the pandemic and the role of DMARDs in COVID-19 treatment.
\end{abstract}

Keywords COVID-19 · DMARD · Disease-modifying anti-rheumatic drugs

\section{Introduction}

The coronavirus disease 2019 (COVID-19) pandemic, caused by severe acute respiratory syndrome coronavirus 2 (SARS$\mathrm{CoV}-2$ ), has been an unprecedented global health crisis, with $>103$ million confirmed cases and $>2.2$ million deaths worldwide as of February 2021 [1]. Patients with autoimmune diseases and their physicians remain concerned regarding potential heightened risks of severe COVID-19 due to immunosuppressive medications needed to control autoimmune disease activity [2]. Meanwhile, glucocorticoids and several diseasemodifying anti-rheumatic drugs (DMARDs), such as hydroxychloroquine and interleukin-6 (IL-6) receptor inhibitors, have been proposed as potential therapies for COVID-19,

This article is part of the Topical Collection on Infections and Arthritis

Zachary S. Wallace

zswallace@mgh.harvard.edu

1 Division of Rheumatology, Allergy, and Immunology, Massachusetts General Hospital, 100 Cambridge Street, 16th Floor, Boston, MA 02114, USA

2 Clinical Epidemiology Program, Mongan Institute, Department of Medicine, Massachusetts General Hospital, Boston, MA, USA

3 Harvard Medical School, Boston, MA, USA and observational studies and clinical trials have revealed variable successes with these agents $[3,4 \cdot 5 \cdot \bullet]$.

In this review, we examine the literature regarding COVID-19 outcomes in patients with rheumatic diseases on glucocorticoids and/or DMARDs at the time of infection (Table 1). We then review the literature regarding the use of glucocorticoids and DMARDs for the treatment of COVID19 , focusing on data from randomized clinical trials (RCTs) (Table 2).

\section{Risk of Severe COVID-19 in Patients on DMARDs}

Early in the COVID-19 pandemic, case series of rheumatic disease patients with COVID-19 reported generally mild clinical courses in patients with inflammatory arthritis, systemic lupus erythematosus, and vasculitis [25, 26, 27]. Early observational studies from longitudinal clinics reported similar incidence of COVID-19 among rheumatic disease patients vs. the general population [28-31]. Although these initial reports were reassuring, a large cohort study of over 17 million patients in the UK (OpenSAFELY) reported higher risk of death from COVID-19 in patients with rheumatoid arthritis, lupus, or psoriasis [32]. Shortly after, comparative cohort studies 


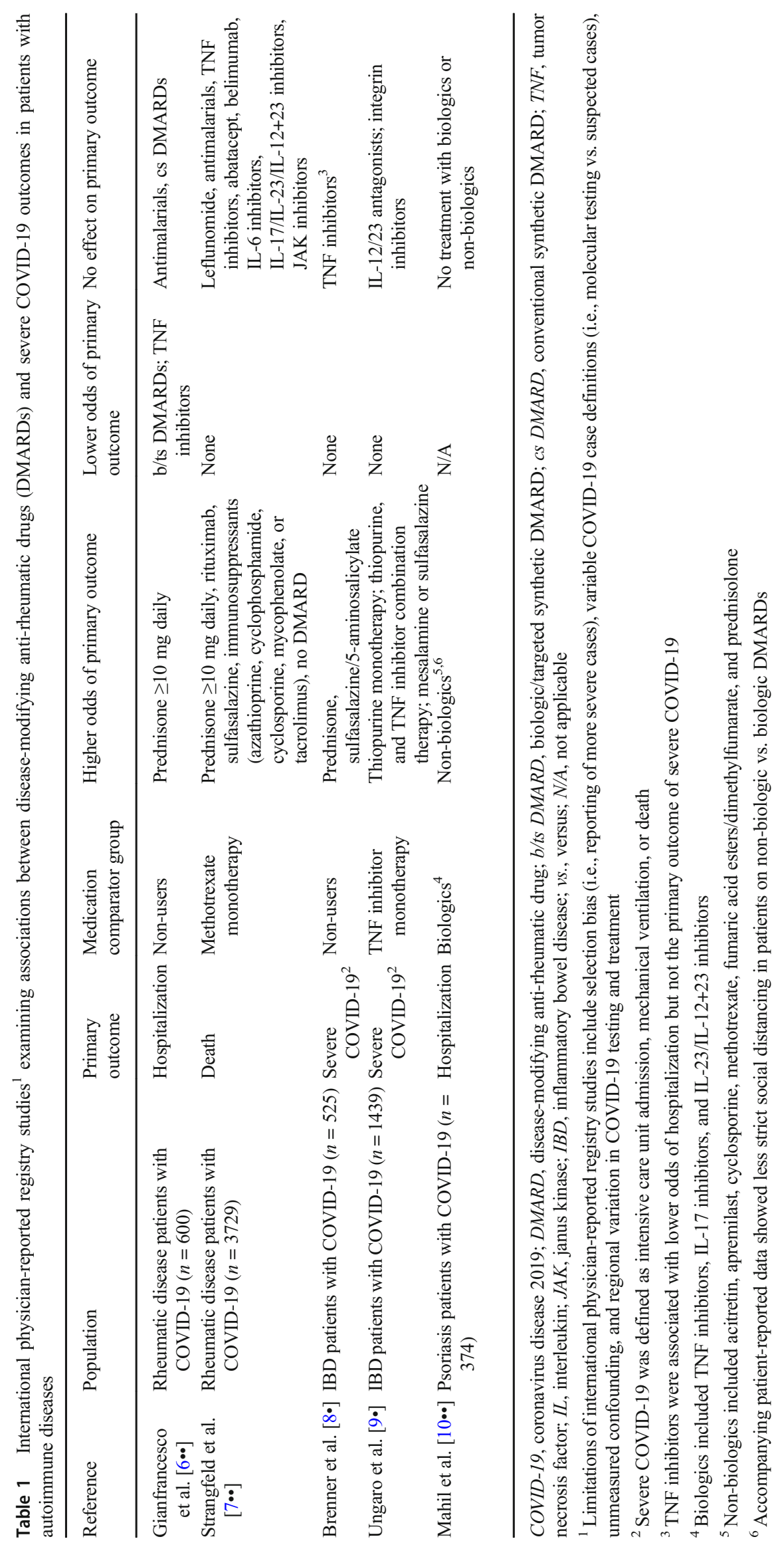




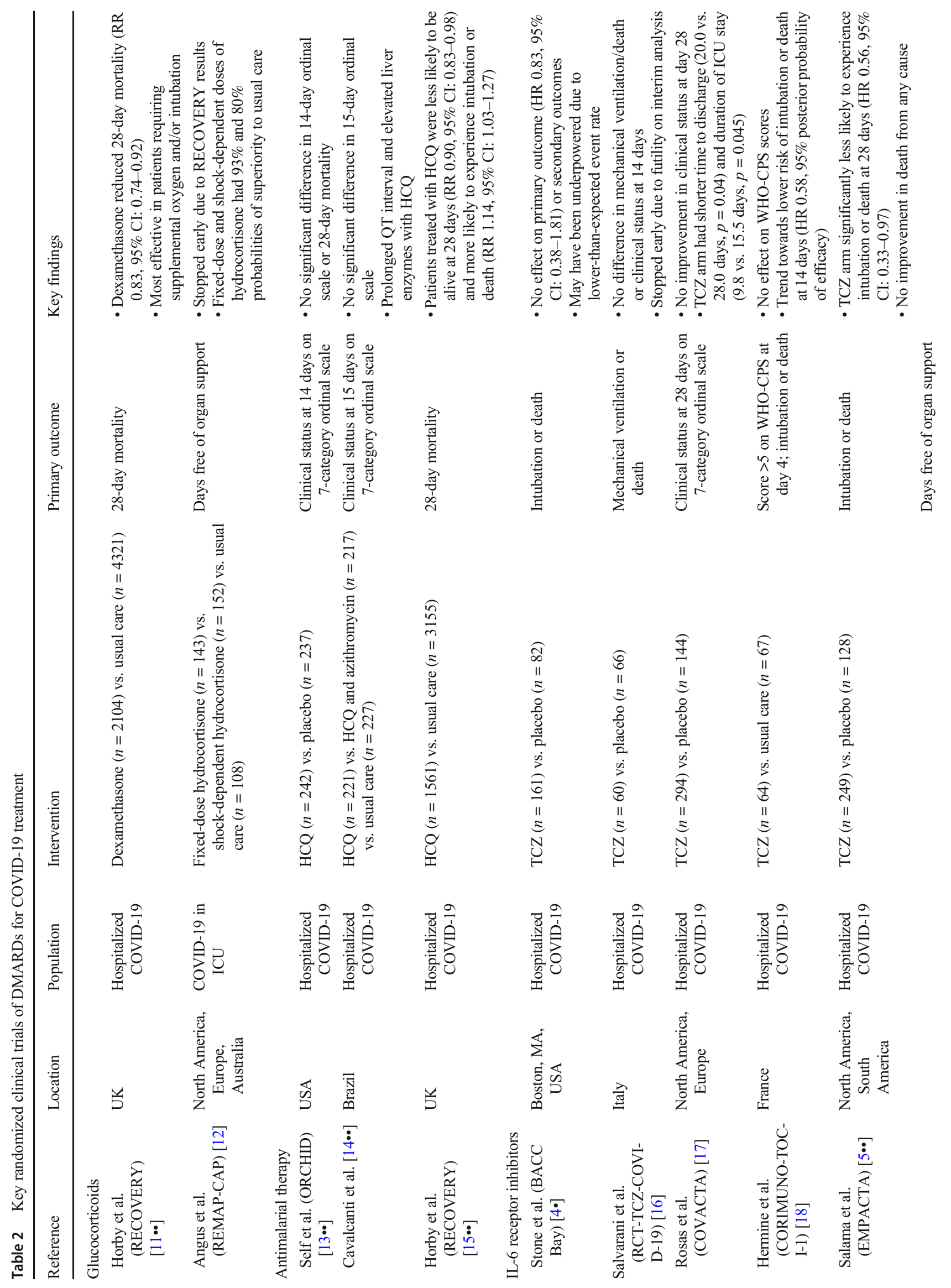




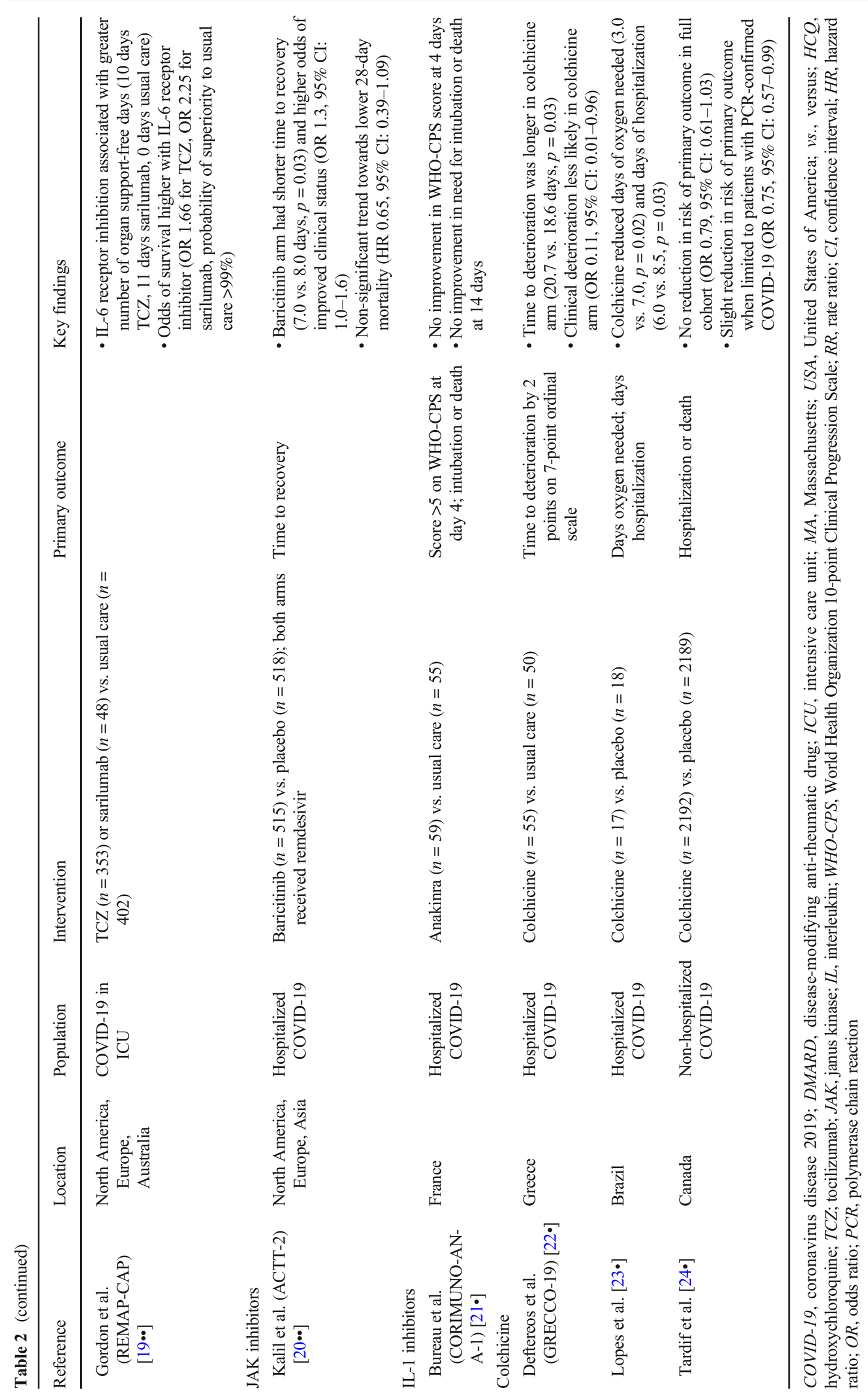


from Wuhan, China, and Boston, MA, reported higher odds of mechanical ventilation in patients with rheumatic diseases vs. comparators without rheumatic diseases [33, 34]. These studies led to heightened concerns that certain risk factors specific to rheumatic disease patients, such as DMARD use, may increase the risk of severe COVID-19 outcomes.

The COVID-19 Global Rheumatology Alliance (GRA) physician-reported registry examined odds of hospitalization among 600 rheumatic disease patients from 40 countries, comparing DMARD users to non-users [6••]. In multivariable models, prednisone doses $\geq 10 \mathrm{mg}$ daily were associated with higher odds of hospitalization (OR $2.05,95 \% \mathrm{CI}: 1.06$ to 3.96$)$ [6••]. Use of conventional synthetic DMARDs, such as methotrexate, azathioprine, or leflunomide, was not associated with higher odds of hospitalization (OR 1.23, 95\% CI: 0.70 to 2.17) [6••]. Interestingly, use of biologic/targeted synthetic DMARDs was associated with lower odds of hospitalization (OR 0.46, 95\% CI: 0.22 to 0.93 ); this latter finding was largely driven by use of tumor necrosis factor (TNF)alpha inhibitors. Mechanistic studies have suggested upregulated TNF-alpha may lead to inflammatory cell death, aberrant germinal center formation, and less robust humoral immune responses in fatal COVID-19, providing a potential biologic rationale for the protective effect of TNF inhibitors, although further study is needed [35, 36].

The GRA conducted a follow-up study of 3729 patients with rheumatic diseases and COVID-19, with notable differences from the first study including a new primary outcome (i.e., COVID-19-related death) and use of an active medication comparator (i.e., methotrexate monotherapy) [7••]. The follow-up study found that prednisone doses $\geq 10 \mathrm{mg}$ daily were associated with higher odds of death (OR 1.69, 95\% CI: 1.18 to 2.41 , consistent with the first study [7••]. Rituximab (OR 4.04, 95\% CI: 2.32 to 7.03), sulfasalazine (OR 3.60, 95\% CI: 1.66 to 7.78), and other immunosuppressants (including azathioprine, cyclophosphamide, cyclosporine, mycophenolate, and tacrolimus; OR 2.22, 95\% CI: 1.43 to 3.46) were associated with higher odds of death compared to methotrexate monotherapy, but there were no higher odds of death with other DMARDs [7••]. Interestingly, patients not on DMARDs also had higher odds of death compared to methotrexate monotherapy (OR 2.11, 95\% CI: 1.48 to 3.01 ) [7••]. Surprisingly, the protective effect associated with TNF inhibitors in the initial study was no longer observed in this followup study. Many of the discrepancies between the first and second GRA studies may be related to the change in the comparator group from non-users to methotrexate monotherapy, so it is challenging to compare the two studies. The second GRA study also used a complex hierarchy of disease and medication groupings, which may limit interpretability and generalizability. However, the second GRA study raises important concerns regarding whether patients on rituximab, sulfasalazine, and certain immunosuppressants are at higher risk of poor outcomes.

The inflammatory bowel disease (IBD) community also established a physician-reported registry, called SECUREIBD, which has been leveraged to examine severe COVID19 outcomes in medication users vs. non-users [8•]. Among 525 patients from 33 countries, risk factors for severe COVID19 included systemic corticosteroids (OR 6.9, 95\% CI: 2.3 to 20.5) and sulfasalazine/5-aminosalicylate (OR 3.1, 95\% CI: 1.3 to 7.7), similar to the GRA findings [8•]. Additionally, the SECURE-IBD registry found that TNF inhibitors were associated with lower risk of COVID-19 hospitalization (OR 0.60, 95\% CI: 0.38 to 0.96 ), similar to the findings of the first GRA study, although there was no effect on the composite outcome of intensive care unit admission, mechanical ventilation, or death (OR 0.90, 95\% CI: 0.37 to 2.17 ) [8•]. The SECUREIBD registry conducted a follow-up study assessing the odds of severe COVID-19 using TNF inhibitor monotherapy as a comparator group [9•]. This study found higher odds of severe COVID-19 with thiopurine monotherapy (OR 4.08, 95\% CI: 1.73 to 9.61 ), combination therapy with a TNF inhibitor and thiopurine (OR 4.01, 95\% CI: 1.65 to 9.78), and mesalamine/ sulfasalazine (OR 3.52, 95\% CI: 1.93 to 6.45) compared to TNF inhibitor monotherapy, providing more granular detail that thiopurines and mesalamine/sulfasalazine may increase risk of poor outcomes [9•].

Lastly, the psoriasis community also established a physician-reported registry, which found that hospitalization was more frequent in patients using non-biologic DMARDs vs. biologic DMARDs (OR 2.84, 95\% CI: 1.31 to 6.18), consistent with the protective effect of biologics observed in the first GRA and SECURE-IBD studies [10••]. However, independent patient-reported data from the psoriasis registry reported stricter social distancing among patients on biologic vs. non-biologic DMARDs, suggesting that adherence to social distancing may be a key mediator in this and other studies [10••]. Although the rheumatology, IBD, and psoriasis registry studies have provided rapid large-scale data on COVID-19 outcomes in patients on DMARDs, these studies are limited by unmeasured confounding and mediators (e.g., adherence to social distancing), selection bias (i.e., physician recall and reporting of more severe cases), variable COVID-19 case definitions, and regional variations in testing and treatment of COVID-19.

In total, evidence from physician-reported registries suggests that patients with autoimmune diseases on glucocorticoids are at higher risk of severe COVID-19 outcomes, while classes of DMARDs (conventional, biologic, and targeted synthetic) do not appear to be associated with higher risk. Some studies suggest that individual DMARDs, such as sulfasalazine, rituximab, and certain immunosuppressants, may be associated with severe COVID-19 outcomes and that TNF inhibitors may protect against severe COVID-19, 
although further studies in non-registry data sets are needed to confirm these findings given discrepancies across registry studies. Importantly, in all the aforementioned studies, COVID-19 risk factors observed in the general population, such as older age and cardiovascular and pulmonary comorbidities, were associated with more severe COVID-19 outcomes; these risk factors may be clinically more important than DMARD use in identifying patients at high risk of severe COVID-19.

Based on the available evidence on DMARDs and COVID-19 to date and prior experiences with infections in rheumatic disease patients, the American College of Rheumatology (ACR) has released guidance regarding the management of rheumatic diseases during the ongoing COVID-19 pandemic [37]. For patients with a known SARS-CoV-2 exposure or confirmed COVID-19, the ACR recommends holding conventional synthetic DMARDs, nonIL-6 biologics, and janus kinase (JAK) inhibitors until 7-14 days after symptom resolution [37]. Hydroxychloroquine may be continued during COVID-19, and patients and providers can use shared decision-making regarding IL-6 receptor inhibitors [37]. Regardless of exposure or infection status, glucocorticoids should be kept at the lowest possible dose [37]. These guidelines will be updated as new data emerge [37].

\section{DMARDs as Therapies for COVID-19}

While the initial stage of COVID-19 is mediated by viral replication, the later stage observed in severe COVID-19 can be associated with a hyperinflammatory syndrome characterized by respiratory and other organ failure, hypercoagulability, and elevated markers of inflammation, including erythrocyte sedimentation rate, C-reactive protein, ferritin, IL-2, IL-6, TNF-alpha, and interferon-gamma (Fig. 1) [38, 39]. While antiviral therapies and monoclonal antibodies to SARS$\mathrm{CoV}-2$ may be the most effective in the initial viral replication phase, anti-inflammatory therapy may alleviate the hyperinflammation that mediates organ failure and death in severe COVID-19 [38]. Given the urgent need to develop treatments for COVID-19, several DMARDs were proposed as repurposed treatments for the hyperinflammatory phase of COVID-19. To date, several drugs commonly used in autoimmune diseases have been tested in RCTs with variable success.

\section{Glucocorticoids}

Glucocorticoids were the first anti-inflammatory medication shown to have a mortality benefit in severe COVID-19. In the RECOVERY trial, a randomized open-label adaptive platform trial, dexamethasone $6 \mathrm{mg}$ (oral or intravenous) once daily for up to 10 days was compared to usual care in patients hospitalized with COVID-19 [11••]. Twenty-eight-day mortality was lower in patients receiving dexamethasone vs. usual care (age-adjusted RR $0.83,95 \%$ CI: 0.74 to 0.92 ) [11••]. Importantly, dexamethasone reduced mortality in patients receiving supplemental oxygen by one-fifth and in patients requiring mechanical ventilation by one-third, but there was no mortality reduction in patients who did not require respiratory support $[11 \bullet \cdot$. This finding suggests that glucocorticoids are effective in patients with severe COVID-19-related respiratory failure but may not have a benefit in patients with early or mild disease.

Further RCTs supported the findings from the RECOVERY trial. In the REMAP-CAP trial, a randomized open-label adaptive platform trial in 121 sites in 8 countries, patients were randomized to a fixed 7-day course of hydrocortisone (50 mg or $100 \mathrm{mg}$ every $6 \mathrm{~h}$ ), shock-dependent course (50 mg every $6 \mathrm{~h}$ when shock was evident), or no hydrocortisone [12]. Although the trial was stopped early due to the release of the RECOVERY results, the fixed-dose and shock-dependent doses of hydrocortisone had $93 \%$ and $80 \%$ probabilities of superiority for the primary endpoint (days free of cardiovascular and/or respiratory support) respectively compared to no hydrocortisone [12]. Furthermore, a meta-analysis of 7 RCTs (total $n=1703$ ) showed reduced mortality in patients receiving corticosteroids vs. those receiving usual care or placebo (summary OR $0.66,95 \% \mathrm{CI}: 0.53$ to 0.82) [40]. Consistent with this evidence, the Infectious Diseases Society of America (IDSA) guidelines recommend dexamethasone in patients with COVID-19 and hypoxemia and/or critical illness [41].

While RCTs overwhelmingly show glucocorticoids are effective in treating severe COVID-19, the registry studies of patients with autoimmune diseases suggested that glucocorticoid use at baseline was associated with higher risk of severe COVID-19. This may be due to confounding by indication in the registry studies, whereby patients with more severe autoimmune disease may be more likely to be on baseline glucocorticoids, and the more severe autoimmune disease may place patients at higher risk of severe COVID-19 outcomes rather than the use of glucocorticoids itself. Alternatively, the timing of glucocorticoid exposure may impact outcomes such that glucocorticoid use during the viral replication phase of COVID-19 may negatively impact the immune response.

\section{Antimalarial Therapy}

Early in vitro data and observational studies suggested that antimalarial drugs such as chloroquine and hydroxychloroquine may be effective against SARS-CoV-2 entry and replication, leading to widespread use, stockpiling, and drug shortages prior to any data from RCTs [3]. However, a meta-analysis of three cohort studies (pooled $n=932$ ) showed no association between hydroxychloroquine use and 


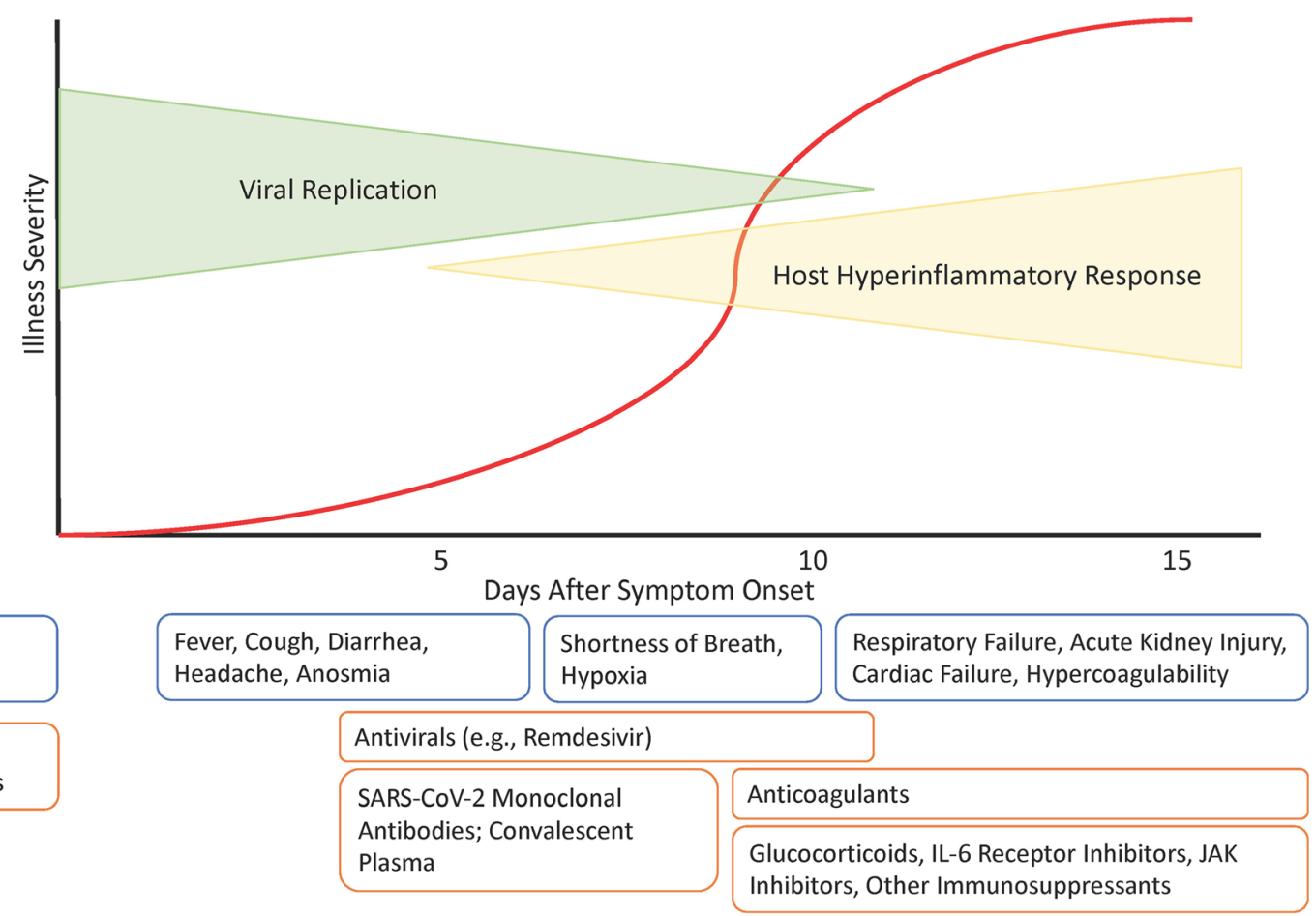

Fig. 1 Illness phases in coronavirus disease 2019 (COVID-19) and potential therapies. Adapted from Siddiqi and Mehra, J Heart Lung Transplant 2020, with permission [38]. SARS-CoV-2, Severe Acute Respiratory Syndrome Coronavirus 2; IL-6, interleukin-6; JAK, janus kinase

mortality, and a meta-analysis of two cohort studies (pooled $n$ = 1549) showed no association between hydroxychloroquine and a composite outcome of invasive mechanical ventilation and mortality [42].

Data from numerous single-center and multicenter RCTs have since confirmed that hydroxychloroquine does not have efficacy in COVID-19 as a prophylactic or therapeutic. RCTs of healthcare workers and others at high risk of SARS-CoV-2 exposure showed no efficacy for hydroxychloroquine for preor post-exposure prophylaxis $[43,44]$. In patients with mild to moderate COVID-19, open-label RCTs of hydroxychloroquine vs. standard of care showed no effect of hydroxychloroquine on reducing viral load or duration until viral clearance [45-47]. A randomized, double-blind, placebo-controlled trial of hydroxychloroquine in symptomatic non-hospitalized adults with COVID-19 showed no difference in severity of symptoms at 14 days [48].

Several large RCTs examined hydroxychloroquine efficacy in hospitalized severe COVID-19. The National Institutes of Health (NIH)-sponsored ORCHID trial randomized hospitalized adults with severe COVID-19 to hydroxychloroquine $(n=242)$ or placebo $(n=237)[13 \bullet \bullet]$. The trial was stopped early due to futility, and there was no improvement in the 7-category COVID-19 ordinal outcome scale at 14 days or mortality at 28 days [13••]. A multicenter RCT of hydroxychloroquine vs. hydroxychloroquine/azithromycin vs. standard of care in Brazil also showed no benefit in moderate/severe hospitalized COVID19 [14••]. Additionally, both hydroxychloroquine regimens were associated with QT prolongation on electrocardiogram (which can trigger life-threatening arrhythmias) and elevated liver enzymes [14••].

Lastly, hydroxychloroquine was examined as a treatment arm in large adaptive platform trials. The World Health Organization SOLIDARITY trial reported no clinical benefit from the use of hydroxychloroquine for hospitalized patients with COVID-19 [49]. The aforementioned RECOVERY trial randomized 1561 hospitalized COVID-19 patients to hydroxychloroquine and 3155 to usual care [15••]. Patients treated with hydroxychloroquine were less likely to be alive at 28 days (RR $0.90,95 \%$ CI: 0.83 to 0.98 ) and more likely to experience invasive mechanical ventilation or death (RR 1.14, 95\% CI: 1.03 to 1.27 ) vs. those receiving usual care, suggesting possible harm from hydroxychloroquine.

There is now abundant evidence from numerous RCTs that hydroxychloroquine does not prevent COVID-19 infection or improve outcomes in mild, moderate, or severe COVID-19, contrary to early observational studies. Hydroxychloroquine has known side effects including QT prolongation, and one of the largest RCTs suggested hydroxychloroquine use may even be associated with lower survival. Therefore, hydroxychloroquine should not be used in the treatment of COVID-19 [41].

\section{IL-6 Receptor Inhibitors}

Initial observational studies from China reported promising results for IL- 6 receptor inhibitors in the treatment of 
COVID-19 [50]. Additionally, a large cohort study of 3924 critically ill patients with COVID-19 in intensive care units (ICUs) in 68 hospitals across the United States (U.S.) showed that treatment with tocilizumab in the first 2 days of ICU admission was associated with a lower risk of death (HR $0.71,95 \%$ CI: 0.56 to 0.92 ) [51].

Although early observational data were promising, early RCTs had negative or equivocal results. RCTs from the U.S. and Italy failed to reach primary and secondary endpoints $[4 \cdot$ 16]. A global multicenter RCT of tocilizumab vs. placebo (COVACTA) in patients hospitalized for severe COVID-19 also failed to reach its primary endpoint, improvement on a 7category ordinal scale, although patients treated with tocilizumab had shorter times to discharge (20.0 vs. 28.0 days, $p$ $=0.04$ ) and duration of ICU stay ( 9.8 vs. 15.5 days, $p=0.045$ ) [17]. An open-label RCT from France (CORIMUNO-TOCI 1) examined tocilizumab vs. usual care in patients with severe COVID-19 requiring $\geq 3 \mathrm{~L} / \mathrm{min}$ of oxygen supplementation [18]. Although this trial showed no effect on the primary outcome (improvement in scores on a clinical progression scale), there was a trend towards lower risk of mechanical ventilation or death at 14 days in the tocilizumab group vs. standard care (median posterior HR $0.58,95 \%$ posterior probability of achieving the predefined efficacy threshold) [18].

Although early trials of tocilizumab had equivocal or negative results, later trials with larger sample sizes and concomitant use of glucocorticoids in the majority $(>80 \%)$ of patients have shown that tocilizumab may be a promising additive treatment for severe COVID-19. The EMPACTA trial, a placebo-controlled RCT of tocilizumab for patients with severe COVID-19, showed that tocilizumab-treated patients were significantly less likely to progress to mechanical ventilation or death (HR 0.56, 95\% CI: 0.33 to 0.97), although there was no improvement in survival alone [5••]. The REMAP-CAP trial of tocilizumab or sarilumab vs. standard care in critically ill COVID-19 patients showed that IL-6 receptor inhibition was associated with significantly greater median organ support-free days and lower mortality compared to standard care [19••]. These two trials suggest that certain patients, especially those who are critically ill in the intensive care unit, may benefit from treatment with tocilizumab in conjunction with glucocorticoids, although the conflicting trial results limit clinical applicability. Currently, the IDSA guidelines recommend against the routine use of tocilizumab in hospitalized COVID-19, although this is a conditional recommendation based on low certainty of evidence and was last updated prior to the availability of the REMAP-CAP results [41].

\section{JAK Inhibitors}

JAK inhibitors may treat the hyperinflammatory phase of COVID-19 by inhibiting several cytokine pathways including
IL-2, IL-6, IL-10, interferon-gamma, and granulocytemacrophage colony-stimulating factor $[20 \bullet, 52,53]$. In addition, among JAK inhibitors, baricitinib (a JAK1/JAK2 oral inhibitor) may block viral entry of SARS-CoV-2 by impairing AP2-associated protein kinase 1 and cyclin G-related kinases, which regulate ACE2 receptor-mediated viral endocytosis $[53,54]$. A double-blind RCT (ACTT-2) of baricitinib vs. placebo on top of background antiviral therapy with remdesivir was conducted in 1033 patients hospitalized with COVID-19 [20••]. Patients treated with baricitinib had shorter time to recovery ( 7 vs. 8 days, $p=0.03$ ) and higher odds of clinical improvement on an 8-category ordinal scale (OR 1.3, 95\% CI: 1.0 to 1.6 ), and there was a non-significant trend towards lower 28-day mortality (HR $0.65,95 \%$ CI: 0.39 to $1.09)$ despite the trial being underpowered to detect mortality as an outcome $[20 \bullet \bullet]$. Notably, there was no higher incidence of thrombosis or secondary infections in the baricitinib-treated $\operatorname{arm}[20 \bullet \cdot]$.

Further studies are needed to evaluate whether baricitinib provides additive benefit to glucocorticoids in patients with severe COVID-19, especially given the cost difference and availability of baricitinib vs. dexamethasone. There are several ongoing phase 2 and 3 clinical trials of baricitinib (NCT04421027, NCT04321993, NCT04340232, NCT04393051), ruxolitinib (NCT04348071, NCT04377620, NCT04334044, NCT04362137), and tofacitinib (NCT04415151, NCT04469114) that may provide answers regarding whether JAK inhibitors provide additional benefits to glucocorticoids and, if so, which patients are most likely to benefit from combination therapy.

\section{IL-1 Inhibitors}

Prior to the COVID-19 pandemic, a secondary analysis of a RCT of anakinra vs. placebo showed that anakinra reduced 28-day mortality in patients with sepsis and features of macrophage activation syndrome; no significant safety signals were observed [55]. Given prior studies in sepsis, IL-1 inhibitors were considered promising DMARDs to repurpose for COVID-19. Additionally, transcriptomic profiling of whole blood from patients with COVID-19 showed elevated IL-1 concentrations prior to the nadir of respiratory function, unlike other pro-inflammatory cytokines, which peaked after respiratory deterioration [56]. Early case series and observational studies supported the hypothesis that IL-1 inhibitors may improve COVID-19 outcomes [57-60].

A French multicenter open-label RCT (CORIMUNOANA-1) of anakinra vs. usual care was conducted among patients hospitalized for COVID-19 requiring $\geq 3 \mathrm{~L} / \mathrm{min}$ of oxygen support [21•]. The trial was stopped early at the recommendation of the data and safety monitoring board; anakinra was not found to improve clinical status at 4 days or need for mechanical ventilation or death at 14 days [21•]. 
However, this trial was conducted prior to the widespread use of remdesivir and dexamethasone, and further studies are needed to determine whether additive therapy with anakinra may improve outcomes. Ongoing phase 2 and 3 clinical trials of anakinra (NCT0443881, NCT04412291, NCT04362111, NCT04357366) and canakinumab (NCT04348448, NCT04362813) may provide further clarity on the role of IL-1 inhibition in severe COVID-19.

\section{TNF Inhibitors}

Anti-TNF therapy has been proposed as a potential treatment for the hyperinflammatory phase of severe COVID-19 because it is overexpressed in patients with severe COVID-19 and associated with poor humoral immune responses in fatal disease [35, 36, 61]. Additionally, patients on baseline TNF inhibitor therapy may have lower odds of severe COVID-19 outcomes in some registry studies, although this may be mediated by other factors such as adherence to social distancing, as previously reviewed $[6 \bullet \bullet, 8 \bullet, 10 \bullet \bullet$. Anti-TNF regimens are currently being investigated in RCTs for COVID-19, including infliximab plus remdesivir (NCT04593940), infliximab (ISRCTN40580903), and adalimumab (ISRCTN33260034, ChiCTR2000030089).

\section{Colchicine}

Colchicine has been proposed as a treatment for COVID19 hyperinflammation due to its ability to inhibit activation of the NLRP3 inflammasome and dampen neutrophil adhesion, migration, and signaling responses [62]. A cohort study from Italy reported that patients treated with colchicine had lower risk of death compared to those treated with usual care (HR $0.15,95 \% \mathrm{CI}$ : 0.06 to 0.37 ) [63]. However, this study used a historical comparator group, which may not be appropriate given the rapidly changing COVID-19 treatment guidelines over time. The Greek Effects of Colchicine in COVID-19 (GRECCO-19) open-label RCT evaluated colchicine vs. usual care in 105 hospitalized COVID-19 patients [22•]. The primary outcome of time to deterioration was longer in the colchicine arm than the usual care arm (20.7 vs. 18.6 days, $p=0.03)$, and clinical deterioration was less likely in the colchicine arm (OR 0.11, 95\% CI: 0.01 to 0.96) [22•]. An interim analysis of a double-blind RCT of colchicine vs. placebo showed that colchicine reduced the duration of hospital stay (6.0 vs. 8.5 days, $p=0.03)$ and need for supplemental oxygen $(53 \%$ vs. $83 \%, p=0.01)$ in patients hospitalized with COVID-19 [23•].

Most recently, a double-blind RCT (COLCORONA) of colchicine $(0.5 \mathrm{mg}$ twice daily for 3 days followed by once daily for 27 days) vs. placebo was conducted in nonhospitalized patients with COVID-19 diagnosed by molecular testing or clinical presentation [24•]. While there was a trend suggesting that colchicine reduced the occurrence of the primary outcome, a composite of hospitalization or death (OR $0.79,95 \%$ CI: 0.61 to 1.03$)$, this did not achieve statistical significance [24•]. When restricted to patients with COVID19 confirmed by molecular testing, there was a statistically significant decrease in the odds of the composite outcome (OR $0.75,95 \%$ CI: 0.57 to 0.99 ), but no statistically significant decrease in the odds of mechanical ventilation (OR 0.50, 95\% CI: 0.23 to 1.07 ) or death (OR $0.56,95 \%$ CI: 0.19 to 1.66) [24•]. Importantly, the trial was terminated early by the investigators due to logistical issues, and therefore it may have been underpowered to detect effects, limiting our ability to apply these findings to non-hospitalized COVID-19 patients in clinical practice [24•].

\section{Conclusions}

In patients with autoimmune diseases, baseline glucocorticoid use is associated with severe COVID-19 outcomes. Given that dexamethasone reduces mortality in severe COVID-19, the finding in patients with autoimmune diseases may be related to the timing of glucocorticoid exposure or confounding by indication, as patients with more severe autoimmune disease are more likely to be on glucocorticoids. Overall, classes of DMARDs including biologic, targeted synthetic, and conventional synthetic DMARDs do not appear to be associated with significantly higher risk of severe COVID-19 outcomes in patients with autoimmune diseases. However, registry studies have raised concern regarding specific medications, such as sulfasalazine, rituximab, and certain immunosuppressants; further studies are needed with non-registry data to replicate these observations.

To date, several RCTs have examined glucocorticoids and DMARDs as treatments for COVID-19. Dexamethasone is associated with reduced mortality in severe COVID-19, especially in patients requiring oxygen supplementation and/or mechanical ventilation. Baricitinib and possibly IL-6 receptor inhibitors may improve outcomes in patients with severe COVID-19; however, further studies are needed to determine which patients are most likely to benefit from these medications as additive therapy to glucocorticoids and antivirals and to develop cost-effective strategies for COVID-19 treatment. Additional RCTs are needed to clarify the efficacy of other DMARDs, including IL-1 inhibitors, TNF inhibitors, and colchicine. There are no RCT data supporting the use of antimalarials for the prevention or treatment of COVID-19, and use of these medications for COVID-19 should be discouraged. The landscape of COVID-19 research is 
rapidly evolving, and treatment recommendations will continue to change over time based on emerging evidence.

Funding KMD is supported by the National Institutes of Health Ruth L. Kirschstein Institutional National Research Service Award (T32-AR007258) and Rheumatology Research Foundation Scientist Development Award. ZSW is supported by NIH/NIAMS (K23AR073334 and L30 AR070520).

\section{Declarations}

Human and Animal Rights and Informed Consent This is a review article and does not present any novel primary data from studies with human or animal subjects performed by the authors.

Conflict of Interest KMD has no disclosures. ZSW reports research support from Bristol-Myers Squibb and Principia and consulting fees from Viela Bio and Medpace.

\section{References}

Papers of particular interest, published recently, have been highlighted as:

- Of importance

•- Of major importance

1. Coronavirus disease 2019 dashboard: World Health Organization; [2-3-21]. Available from: https://covid19.who.int/.

2. Michaud K, Wipfler K, Shaw Y, Simon TA, Cornish A, England $\mathrm{BR}$, et al. Experiences of patients with rheumatic diseases in the US during early days of the COVID-19 pandemic. ACR Open Rheumatol. 2020;2(6):335-43.

3. Sattui SE, Liew JW, Graef ER, Coler-Reilly A, Berenbaum F, Duarte-García A, et al. Swinging the pendulum: lessons learned from public discourse concerning hydroxychloroquine and COVID-19. Expert Rev Clin Immunol. 2020;16(7):659-66.

4. Stone JH, Frigault MJ, Serling-Boyd NJ, Fernandes AD, Harvey L, Foulkes AS, et al. Efficacy of tocilizumab in patients hospitalized with COVID-19. N Engl J Med. 2020;383:2333-44 This early RCT of tocilizumab vs. placebo in hospitalized COVID-19 showed no effect of tocilizumab on COVID-19 outcomes.

5.• Salama C, Han J, Yau L, Reiss WG, Kramer B, Neidhart JD, et al. Tocilizumab in patients hospitalized with COVID-19 pneumonia. N Engl J Med. 2021;384:20-30 This RCT of tocilizumab vs. placebo in patients hospitalized with COVID-19 showed lower risk of intubation or death at 28 days in the tocilizumab-treated arm.

6.• Gianfrancesco MA, Hyrich KL, Al-Adely S, Carmona L, Danila MI, Gossec L, et al. Characteristics associated with hospitalization for COVID-19 in people with rheumatic disease: data from the COVID-19 Global Rheumatology Alliance physician-reported registry. Ann Rheum Dis. 2020;79:859-66 In this study from the Global Rheumatology Alliance physician-reported registry, prednisone doses above $10 \mathrm{mg}$ daily were associated with higher odds of hospitalization in rheumatic disease patients with COVID-19, while biologic/targeted synthetic DMARDs and TNF inhibitors were associated with lower odds of hospitalization.
7.• Strangfeld A, Schäfer M, Gianfrancesco MA, Lawson-Tovey S, Liew JW, Ljung L, et al. Factors associated with COVID-19related death in people with rheumatic diseases: results from the COVID-19 Global Rheumatology Alliance physician-reported registry. Ann Rheum Dis. 2021;Advance online publication. In this follow-up study from the Global Rheumatology Alliance physician-reported reigstry, rheumatic disease patients on rituximab, sulfasalazine, or certain immunosuppressants (including azathioprine, cyclophosphamide, cyclosporine, mycophenolate, or tacrolimus) had higher odds of death than comparators on methotrexate monotherapy.

8. Brenner EJ, Ungaro RC, Gearry RB, Kaplan GG, Kissous-Hunt M, Lewis JD, et al. Corticosteroids, but not TNF antagonists, are associated with adverse COVID-19 outcomes in patients with inflammatory bowel disease: results from an international registry. Gastroenterology. 2020;159(2):481-91 In a physician-reported registry of inflammatory bowel disease patients with COVID19, prednisone and sulfasalazine were associated with higher odds of severe COVID-19 compared to non-users of these medications.

9. Ungaro RC, Brenner EJ, Gearry RB, Kaplan GG, Kissous-Hunt M, Lewis JD, et al. Effect of IBD medications on COVID-19 outcomes: results from an international registry. Gut. 2020;Advance online publication. In a follow-up study of inflammatory bowel disease patients with COVID-19 in a physician-reported registry, thiopurines and mesalamine/sulfasalazine were associated with higher odds of severe COVID-19 compared to TNF inhibitor monotherapy.

10.• Mahil SK, Dand N, Mason KJ, ZZN Y, Tsakok T, Meynell F, et al. Factors associated with adverse COVID-19 outcomes in patients with psoriasis - insights from a global registry-based study. J Allergy Clin Immunol. 2020;147(1):60-71 In a physicianreported registry of psoriasis patients with COVID-19, patients on non-biologics had higher odds of hospitalization compared to patients on biologics. Importantly, accompanying patientreported data showed greater adherence to social distancing measures in patients on biologics.

11.• Horby PW, Lim WS, Emberson JR, Mafham M, Bell JL, Linsell L, et al. Dexamethasone in hospitalized patients with COVID-19: preliminary report. N Engl J Med. 2020;Advance online publication. This large RCT of dexamethasone vs. usual care was the first study to report that glucocorticoids lowered the risk of mortality in patients hospitalized with COVID-19.

12. Angus DC, Derde L, Al-Beidh F, Annane D, Arabi Y, Beane A, et al. Effect of hydrocortisone on mortality and organ support in patients with severe COVID-19. JAMA. 2020;324(13):1317-29.

13.• Self WH, Semler MW, Leither LM, Casey JD, Angus DC, Brower $\mathrm{RG}$, et al. Effect of Hydroxychloroquine on clinical status at 14 days in hospitalized patients with COVID-19. JAMA. 2020;324(21):2165-76 This RCT of hydroxychloroquine vs. placebo in patients hospitalized with COVID-19 showed that hydroxychloroquine did not improve clinical status or mortality.

14.• Cavalcanti AB, Zampieri FG, Rosa RG, Azevedo LCP, Veiga VC, Avezum A, et al. Hydroxychloroquine with or without azithromycin in mild-to-moderate COVID-19. N Engl J Med. 2020;383(21):2041-52 This RCT of hydroxychloroquine vs. hydroxychloroquine and azithromycin vs. usual care in patients hospitalized with COVID-19 showed no improvement in clinical status with either hydroxychloroquine regimen. Additionally, both hydroxychloroquine regimens were associated with QT prolongation and elevated liver enzymes.

15.• RECOVERY Collaborative Group. Effect of Hydroxychloroquine in Hospitalized Patients with Covid-19. N Engl J Med. 2020;383(21):2030-40 This RCT of hydroxychloroquine vs. usual care in patients hospitalized with COVID-19 showed that 
patients treated with hydroxychloroquine were more likely to experience intubation or death compared to those receiving usual care.

16. Salvarani C, Dolci G, Massari M, Merlo DF, Cavuto S, Savoldi L, et al. Effect of tocilizumab vs standard care on clinical worsening in patients hospitalized with COVID-19 pneumonia. JAMA Intern Med. 2021;181(1):24-31.

17. Rosas I, Bräu N, Waters M, Go RC, Hunter BD, Bhagani S, et al. Tocilizumab in hospitalized patients with COVID-19 pneumonia. medRxiv. 2020;Advance online publication.

18. Hermine O, Mariette X, Tharaux P-L, Resche-Rigon M, Porcher R, Ravaud P. Effect of tocilizumab vs usual care in adults hospitalized with COVID-19 and moderate or severe pneumonia. JAMA Intern Med. 2021;181(1):32-40.

19.• Gordon AC, Mouncey PR, Al-Beidh F, Rowan KM, Nichol AD, Arabi YM, et al. Interleukin-6 receptor antagonists in critically ill patients with Covid-19 - preliminary report. medRxiv. 2021;Advance online publication. This RCT of tocilizumab or sarilumab vs. usual care in patients with COVID-19 requiring intensive care showed a greater number of organ support-free days and greater survival in the arm receiving IL-6 receptor inhibitor therapy.

20.• Kalil AC, Patterson TF, Mehta AK, Tomashek KM, Wolfe CR, Ghazaryan V, et al. Baricitinib plus remdesivir for hospitalized adults with COVID-19. N Engl J Med. 2020;Advance online publication. This RCT of baricitinib vs. placebo on background remdesivir therapy in hospitalized COVID-19 showed shorter time to recovery in the baricitinib-treated arm.

21. Bureau S, Dougados M, Tibi A, Azoulay E, Cadranel J, Emmerich $\mathrm{J}$, et al. Effect of anakinra versus usual care in adults in hospital with COVID-19 and mild-to-moderate pneumonia (CORIMUNOANA-1): a randomised controlled trial. Lancet Respir Med. 2021;Advance online publication. This RCT of anakinra vs. usual care in patients hospitalized with COVID-19 showed no effect on clinical status or a composite outcome of intubation or death.

22. Deftereos SG, Giannopoulos G, Vrachatis DA, Siasos GD, Giotaki SG, Gargalianos P, et al. Effect of colchicine vs standard care on cardiac and inflammatory biomarkers and clinical outcomes in patients hospitalized with coronavirus disease 2019. JAMA Netw Open. 2020;3(6):1-14 This RCT of colchicine vs. usual care in patients hospitalized with COVID-19 showed longer time to deterioration in the colchicine-treated arm.

23. Lopes MIF, Bonjorno LP, Giannini MC, Amaral NB, Benatti MN, Rezek UC, et al. Beneficial effects of colchicine for moderate to severe COVID-19: an interim analysis of a randomized, doubleblinded, placebo controlled clinical trial. medRxiv. 2020;Advance online publication. This interim analysis of a RCT of colchicine vs. placebo showed reduced days of oxygen needed and days of hospitalization in the colchicine-treated arm.

24. Tardif J-C, Bouabdallaoui N, L'Allier PL, Gaudet D, Shah B, Pillinger MH, et al. Efficacy of colchicine in non-hospitalized patients with COVID-19. medRxiv. 2021;Advance online publication. This RCT of colchicine vs. placebo in non-hospitalized COVID-19 showed a trend towards reduced risk of hospitalization or death in the colchicine-treated arm, although the trial was stopped early due to logistical reasons, limiting interpretability.

25. Haberman R, Axelrad J, Chen A, Castillo R, Yan D, Izmirly P, et al. COVID-19 in immune-mediated inflammatory diseases - case series from New York. N Engl J Med. 2020;383:85-8.

26. Monti S, Balduzzi S, Delvino P, Bellis E, Quadrelli VS, Montecucco C. Clinical course of COVID-19 in a series of patients with chronic arthritis treated with immunosuppressive targeted therapies. Ann Rheum Dis. 2020;79(5):667-8.
27. Tomelleri A, Sartorelli S, Campochiaro C, Baldissera EM, Dagna L. Impact of COVID-19 pandemic on patients with large-vessel vasculitis in Italy: a monocentric survey. Ann Rheum Dis. 2020;79(9):1252-3.

28. Favalli EG, Ingegnoli F, Cimaz R, Caporali R. What is the true incidence of COVID-19 in patients with rheumatic diseases? Ann Rheum Dis. 2021;80(2):1.

29. Emmi G, Battiol A, Mattioli I, Silvestri E, Di Scala G, Urban ML, et al. SARS-CoV-2 infection among patients with systemic autoimmune diseases. Autoimmun Rev. 2020;19:1-5.

30. Michelena X, Borrell H, Lopez-Corbeto M, Lopez-Lasanta M, Moreno E, Pascual-Pastor M, et al. Incidence of COVID-19 in a cohort of adult and pediatric patients with rheumatic diseases treated with targeted biologic and synthetic disease-modifying antirheumatic drugs. Semin Arthritis Rheum. 2020;50:564-70.

31. Conticini E, Bargagli E, Bardelli M, Rana GD, Baldi C, Cameli P, et al. COVID-19 pneumonia in a large cohort of patients treated with biological and targeted synthetic antirheumatic drugs. Ann Rheum Dis. 2021;80(0):1-2.

32. Williamson E, Walker AJ, Bhaskaran KJ, Bacon S, Bates C, Morton CE, et al. OpenSAFELY: factors associated with COVID-19-related hospital death in the linked electronic health records of 17 million adult NHS patients. Nature. 2020;584:430-6.

33. Ye C, Cai S, Shen G, Guan H, Zhou L, Hu Y, et al. Clinical features of rheumatic patients infected with COVID-19 in Wuhan, China. Ann Rheum Dis. 2020;79:1007-13.

34. D'Silva KM, Serling-Boyd N, Wallwork R, Hsu TYT, Fu X, Gravallese EM, et al. Clinical characteristics and outcomes of patients with coronavirus disease 2019 (COVID-19) and rheumatic disease: a comparative cohort study from a United States "hot spot". Ann Rheum Dis. 2020;79:1156-62.

35. Kaneko N, Kuo H-H, Boucau J, Farmer JR, Allard-Chamard H, Mahajan VS, et al. Loss of Bcl-6-expressing T follicular helper cells and germinal centers in COVID-19. Cell. 2020;183:1-15.

36. Karki R, Sharma BR, Tuladhar S, Williams EP, Zalduondo L, Samir P, et al. Synergism of TNF- $\alpha$ and IFN- $\gamma$ triggers inflammatory cell death, tissue damage, and mortality in SARS-CoV-2 infection and cytokine shock syndromes. Cell. 2021;184(1):149-68.

37. Mikuls TR, Johnson SR, Fraenkel L, Arasaratnam RJ, Baden LR, Bermas BL, et al. American College of Rheumatology guidance for the management of rheumatic disease in adult patients during the covid-19 pandemic: version 2. Arthritis Rheumatol. 2020;72(9):e1e12.

38. Siddiqi HK, Mehra MR. COVID-19 illness in native and immunosuppressed states: a clinical-therapeutic staging proposal. J Heart Lung Transplant. 2020;39(5):405-7.

39. Lu C, Li S, Liu Y. Role of immunosuppressive therapy in rheumatic diseases concurrent with covid-19. Ann Rheum Dis. 2020;79:7379.

40. Sterne JAC, Murthy S, Diaz JV, Slutsky AS, Villar J, Angus DC, et al. Association between administration of systemic corticosteroids and mortality among critically ill patients with COVID-19. JAMA. 2020;324(13):1330-41.

41. Bhimraj A, Morgan RL, Shumaker AH, Lavergne V, Baden LR, Cheng VC, et al. Infectious Diseases Society of America guidelines on the treatment and management of patients with COVID-192021 [2-4-21]. Available from: https://www.idsociety.org/practiceguideline/covid-19-guideline-treatment-and-management/. Accessed 4 Feb 2021.

42. Putman M, Chock YPE, Tam H, Kim AHJ, Sattui SE, Berenbaum F, et al. Antirheumatic disease therapies for the treatment of COVID-19: a systematic review and meta-analysis. Arthritis Rheum. 2020; Advance online publication.

43. Boulware DR, Pullen MF, Bangdiwala AS, Pastick KA, Lofgren $\mathrm{SM}$, Okafor EC, et al. A randomized trial of hydroxychloroquine as 
postexposure prophylaxis for COVID-19. N Engl J Med. 2020;383(6):517-25.

44. Abella BS, Jolkovsky EL, Biney BT, Uspal JE, Hyman MC, Frank I, et al. Efficacy and safety of hydroxychloroquine vs placebo for pre-exposure SARS-CoV-2 prophylaxis among health care workers. JAMA Intern Med. 2020; Advance online publication.

45. Tang W, Cao Z, Han M, Wang Z, Chen J, Sun W, et al. Hydroxychloroquine in patients with mainly mild to moderate coronavirus disease 2019: open label, randomised controlled trial. BMJ. 2020;369:1-11.

46. Mitjà $\mathrm{O}$, Corbacho-Monné $\mathrm{M}$, Ubals $\mathrm{M}$, Tebe $\mathrm{C}$, Peñafiel $\mathrm{J}$, Tobias A, et al. Hydroxychloroquine for early treatment of adults with mild COVID-19: a randomized-controlled trial. Clin Infect Dis. 2020; Advance online publication.

47. Mitjà $\mathrm{O}$, Corbacho $\mathrm{M}, \mathrm{G}-$ Beiras $\mathrm{C}$, Tebé $\mathrm{C}$, Tobias $\mathrm{A}$, Ballana $\mathrm{E}$, et al. Hydroxychloroquine alone or in combination with cobicistatboosted darunavir for treatment of mild COVID-19: A ClusterRandomized Clinical Trial. SSRN Electron J. 2020; Advance online publication.

48. Skipper CP, Pastick KA, Engen NW, Bangdiwala AS, Abassi M, Lofgren SM, et al. Hydroxychloroquine in nonhospitalized adults with early COVID-19. Ann Intern Med. 2020;173(8):623-31.

49. WHO Solidarity Trial Consortium. Repurposed antiviral drugs for Covid-19 - Interim WHO Solidarity Trial Results. N Engl J Med. 2020; Advance Online Publication.

50. Luo P, Liu Y, Qiu L, Liu X, Liu D, Li J. Tocilizumab treatment in COVID-19: a single center experience. J Med Virol. 2020;92(7): 814-8.

51. Gupta S, Wang W, Hayek SS, Chan L, Mathews KS, Melamed ML, et al. Association between early treatment with tocilizumab and mortality among critically ill patients with COVID-19. JAMA Intern Med. 2021;181(1):41-51.

52. Richardson P, Griffin I, Tucker C, Smith D, Oechsle O, Phelan A, et al. Baricitinib as potential treatment for 2019-nCoV acute respiratory disease. Lancet. 2020;395(10223):e30-1.

53. Amigues I, Pearlman AH, Patel A, Reid P, Robinson PC, Sinha R, et al. Coronavirus disease 2019: investigational therapies in the prevention and treatment of hyperinflammation. Expert Rev Clin Immunol. 2020;16(12):1185-204.
54. Zhong J, Tang J, Ye C, Dong L. The immunology of COVID-19: is immune modulation an option for treatment? Lancet Rheumatol. 2020;2(7):e428-e36.

55. Shakoory B, Carcillo JA, Chatham WW, Amdur RL, Zhao H, Dinarello CA, et al. Interleukin-1 receptor blockade is associated with reduced mortality in sepsis patients with features of macrophage activation syndrome. Crit Care Med. 2016;44(2):275-81.

56. Ong EZ, Chan YFZ, Leong WY, Lee NMY, Kalimuddin S, Haja Mohideen SM, et al. A dynamic immune response shapes COVID19 progression. Cell Host Microbe. 2020;27(6):879-82.

57. Navarro-Millán I, Sattui SE, Lakhanpal A, Zisa D, Siegel CH, Crow MK. Use of anakinra to prevent mechanical ventilation in severe COVID-19: a case series. Arthritis Rheum. 2020;72(12): 1990-7.

58. Balkhair A, Al-Zakwani I, Al Busaidi M, Al-Khirbash A, Al Mubaihsi S, Bataher H, et al. Anakinra in hospitalized patients with severe COVID-19 pneumonia requiring oxygen therapy: results of a prospective, open-label, interventional study. Int J Infect Dis. 2021;103:288-96.

59. Kooistra EJ, Waalders NJB, Grondman I, Janssen NAF, De Nooijer $\mathrm{AH}$, Netea MG, et al. Anakinra treatment in critically ill COVID-19 patients: a prospective cohort study. Crit Care. 2020;24(1):688-99.

60. Huet T, Beaussier H, Voisin O, Jouveshomme S, Dauriat G, Lazareth I, et al. Anakinra for severe forms of COVID-19: a cohort study. Lancet Rheumatol. 2020;2(7):393-400.

61. Robinson PC, Liew DFL, Liew JW, Monaco C, Richards D, Shivakumar S, et al. The potential for repurposing anti-TNF as a therapy for the treatment of COVID-19. Med. 2020;1(1):90-102.

62. Reyes AZ, Hu KA, Teperman J, Wampler Muskardin TL, Tardif JC, Shah B, et al. Anti-inflammatory therapy for COVID-19 infection: the case for colchicine. Ann Rheum Dis. 2020; Advance online publication.

63. Scarsi M, Piantoni S, Colombo E, Airó P, Richini D, Miclini M, et al. Association between treatment with colchicine and improved survival in a single-centre cohort of adult hospitalised patients with COVID-19 pneumonia and acute respiratory distress syndrome. Ann Rheum Dis. 2020; Advance online publication.

Publisher's Note Springer Nature remains neutral with regard to jurisdictional claims in published maps and institutional affiliations. 